\title{
Self-Management Framework for Mobile Autonomous Systems
}

\author{
E. Asmare • A. Gopalan • M. Sloman • \\ N. Dulay • E. Lupu
}

the date of receipt and acceptance should be inserted later

\begin{abstract}
The advent of mobile and ubiquitous systems has enabled the development of autonomous systems such as wireless-sensors for environmental data collection and teams of collaborating Unmanned Autonomous Vehicles (UAVs) used in missions unsuitable for humans. However, with these range of new application-domains come a new challenge - enabling self-management in mobile autonomous systems. Autonomous systems have to be able to manage themselves individually as well as to form self-managing teams which are able to recover or adapt to failures, protect themselves from attacks and optimise performance.

This paper proposes a novel distributed policy-based framework that enables autonomous systems of varying scale to perform self-management individually and as a team. The framework allows missions to be specified in terms of roles in an adaptable and reusable way, enables dynamic and secure team formation with a utility-based approach for optimal role assignment, caters for communication link maintenance amongst team-members and recovery from failure. Adaptive management is achieved by employing a policy-based architecture to enable dynamic modification of the management strategy relating to resources, role behaviour, communications and team management, without interrupting the basic software within the system.

Evaluation of the framework shows that it is scalable with respect to the number of roles, and consequently the number of autonomous systems participating in the mission. It is also shown to be optimal with respect to role assignments, and robust to intermittent communication link disconnections and permanent team-member failures.
\end{abstract}

Keywords Autonomic management, collaborating autonomous vehicles, mission management, communication failure recovery.

Department of Computing, Imperial College London

180 Queen's Gate, London, SW7 2RH, UK E-mail: \{e.asmare, a.gopalan, m.sloman, n.dulay, e.c.lupu\}@imperial.ac.uk 


\section{Introduction}

Technological advances in engineering and communication have paved the way for increasing use of autonomous systems. Given high level objectives from administrators an autonomic computing system is able to self-manage. It is also able to simplify the task of managing today's complex computing systems [16, 22]. Self-managing autonomous systems have the potential for providing the information needed to assist rescue operations - by locating survivors, identifying affected areas, and organising the collaborative efforts of the response team members. They also find uses in disaster management, including earthquakes, forest fires and floods, unmanned vehicles and military applications. All scenarios listed above require groups of entities that are able to communicate using a wireless ad-hoc network. In this paper, we focus on teams of unmanned autonomous vehicles, since this is the testbed that we have been using.

Unmanned Autonomous Vehicle (UAV) is a type of robot that is often used for applications such as those listed above. A challenge in using UAVs for these applications is enabling self-management so that they can accomplish a mission autonomously without human intervention. This means that UAVs need to adapt their behaviour to current context, location, activity, available resources such as battery power and available services such as quality of communication links. They should be self-managing in that they have to recover or adapt to component failures and optimise performance to best utilise available resources. Additionally, a team of UAVs should cooperate to achieve a particular mission, such as surveillance of a specific area. Collaborating UAVs form a Self Managed Cell (SMC) [30], the general autonomic computing [22] architectural principle we use for realising self-management of individual and teams of UAVs.

An SMC consists of multiple UAVs and at least one commander, which could be a human or another UAV, and which effectively controls the group. The commander role could be reassigned to a backup UAV in case the primary commander fails. The commander is provided with a mission specification by its command base and assembles the required UAVs to perform the mission. The mission specification [13] defines how specific roles are assigned to UAVs based on their credentials and capabilities, as well as when and how to adapt the mission to changes in context or failures. The adaptive management of UAVs is achieved using policy-based techniques that allow dynamic modification of the management strategy relating to resources, task behaviour, communications and team management, without reloading the basic software within the UAV. SMCs can be combined in peer-to-peer or composition relationships to reflect complex collaborations between multiple teams to achieve an overall mission [36].

The mission specification also defines a role management hierarchy and the behaviour of these roles in terms of policies specified using the Ponder2 [35] policy framework. Ponder2 is a generic object management system supporting dynamic loading, unloading, enabling and disabling of active managed objects 
capable of receiving action commands and performing actions. It supports obligation policies (event-condition-action rules) to trigger specific actions to be performed when an event, such as the discovery of a new UAV occurs. Authorisation policies specify conditions under which services and resources within a UAV can be accessed by other UAVs performing a specific role. Policies are interpreted, hence they can be dynamically loaded, enabled or disabled at run-time without shutting down a system in order to adapt the management strategy. When a mission is instantiated the commander will download its role behaviour specifications (the policies) and start the mission. When a new UAV comes into the communication range, the commander checks the UAV's security credentials. If these are satisfactory, the UAV will be allocated to one or more roles based on its capabilities and may be given a subset of the mission roles to manage. The new UAVs may in turn allocate a subset of its mission roles to other UAVs and the whole process finally results in a formation of a failure-tolerant management tree which facilitates control and state information collection.

To ensure effective completion of the mission, the available resources must be used efficiently. When forming a dynamic team, if the assignment of roles to UAVs is first-come-first-served - where any capable UAV is immediately assigned to an available role, the team may end up in a state where more capable UAVs are assigned to less demanding roles. This may result in a UAV with specific capabilities being assigned to a role that could have been fulfilled by any of the UAVs and the role requiring the specific capability remains unfulfilled, resulting in an incomplete team. It is thus necessary to perform optimisation on the set of discovered UAVs and the mission roles in order to best utilise the available resources as first-come-first-served is suboptimal.

This paper extends the architecture presented in $[4,13,14]$ by elaborating the different components of the framework and presenting an optimisation scheme that optimally assigns available UAVs to roles based on their capabilities. The scheme considers both local assignments by the commander and global future assignments by other team members, so that the team will reach its optimal configuration provided UAVs with the necessary capabilities are available. The proposed optimisation scheme is modelled and evaluated and the results show that the scheme significantly improves the role assignment.

Although we focus on UAV based applications in this paper, the concepts apply to any application of mobile wireless devices collaborating via ad-hoc networks and performing various roles within a team. In this paper, we do not focus on the lower level sensing capabilities of UAVs, i.e. the faults and uncertainties arising from sensors. There is other work in our group dealing with policy-based fault detection and recovery in sensor networks [31].

The rest of the paper is organised as follows. In Section 2, we will present the overview of the architecture. Section 3 details our mission specification approach. Section 4 details the dynamic team formation approach in accordance with the mission specification. Section 5 presents the performance evaluation of the framework. We present related work in Section 6 followed by conclusions. 


\section{Overview of Self-management Architecture}

In real-life applications, multiple autonomous vehicles deployed in a mission collaborate and use services or resources from each other. To be able to form and use a dynamic collaborative team of autonomous systems in accordance with a high level mission specification, a method for describing the capabilities of autonomous systems, discovering available heterogeneous autonomoussystems, securely admitting them to the team, assigning them to an appropriate role based on their capability and maintaining the team is necessary. The adaptive, policy-based self-management architecture is comprised of mission, team and communication layers. Fig. 1 illustrates the layers and interactions among them. We will briefly detail the scheme used for securely admitting an UAV to the team, while Sections 3 and 4 focus on mission management and team management respectively.

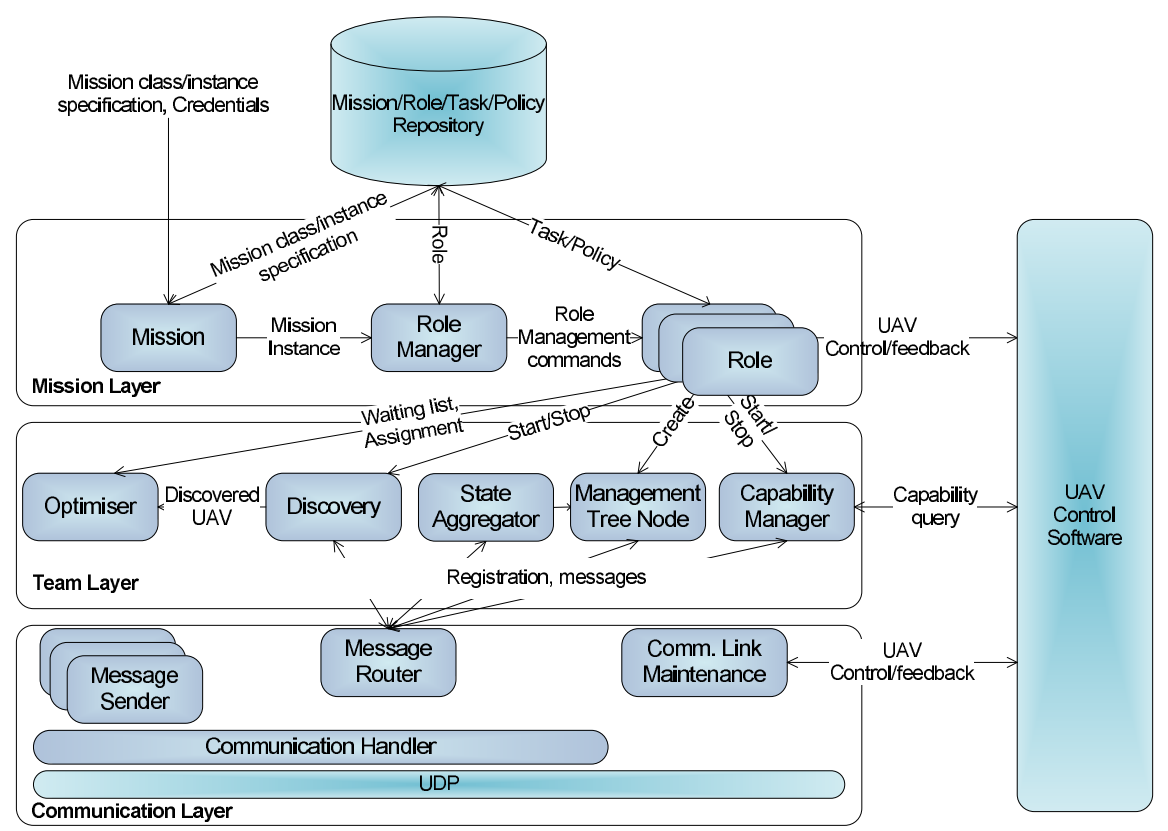

Fig. 1 Self-management Architecture

UAVs that are part of a mission can change dynamically over time with new UAVs joining or leaving the group. These new UAVs may also belong to other organisations (e.g allies). The degree to which they are trusted may depend on the organisation to which they belong. Authenticating an UAV before it joins the SMC (or team) and protecting the ensuing group communication is thus necessary to ensure the credibility of the mission. Therefore, all SMCs provide 
the required security mechanisms to perform authentication, encryption and access control.

Consider the scenario depicted in Fig. 2. $U A V_{1}, U A V_{2}$ and commander $C D_{1}$ form the Self Managed Cell $S M C_{1}$, while $U A V_{3}, U A V_{4}, U A V_{5}$ and commander $C D_{2}$ form $S M C_{2}$ and $U A V_{6}, U A V_{7}$ and commander $C D_{3}$ form $S M C_{3}$. $S M C_{1}$ and $S M C_{2}$ belong to the same organisation $\left(H Q_{1}\right)$, while $S M C_{3}$ belongs to another organisation $\left(H Q_{2}\right)$. We assume the coalition between different organisations is achieved by using a Central Command Centre $\left(C^{3}\right)$ and use the Certificate Public Key Infrastructure (C-PKI) [17] to ensure authentication, confidentiality and message integrity. The system assumes a single certification authority $\left(C^{3}\right)$, which issues certified public/private keys to all UAVs in the mission and maintains a Certificate Revocation List (CRL). The C-PKI system is also used to exchange a common secret key generated using the Diffie-Hellman protocol [12] between each member of the team and the commander (more details on the Security protocol can be found in [14]).

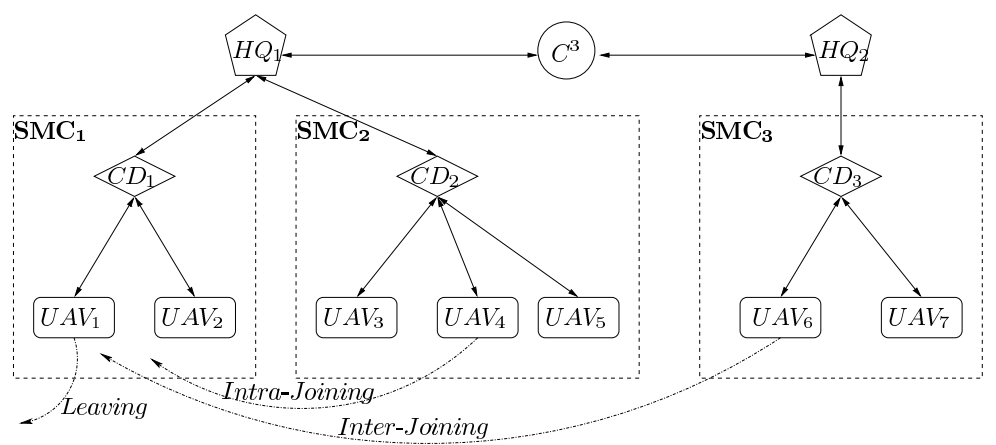

Fig. 2 Example of UAVs forming Self Managed Cells belonging to different organisations

\section{Mission Management}

A mission for a team of UAVs is specified in terms of roles. The mission specification defines how UAVs will be assigned to perform specific roles within the team, based on their capabilities, as well as when and how to adapt the mission to changes in context or failures. This specification will then be loaded onto a UAV which has the necessary capabilities for managing the mission and consequently assumes the commander role. The commander uses its discovery service to discover and authenticate UAVs within its communication range. The discovered UAVs provide their capability descriptions to the commander which checks them against the capability requirements of available roles and perform optimal assignments. The assigned UAVs recursively repeat this process if their mission includes managing other UAVs, as a result creating a tree with the commander UAV as the root. This tree is used to communi- 
cate management messages, collect state information and organise the roles hierarchically each with a unique identity so as to make the team robust and capable of recovering from failure (details of tree formation and maintenance are given in [14]).

To illustrate our approach, we consider an example mission to determine whether an area is safe for humans. The following main roles are identified: Commander $(C)$ : controls the mission and allocates UAVs to roles. Surveyor $(S)$ : explores the house and builds a map. Hazardous material detector (H). Communication relay $(R)$ : maintains communication among UAVs by forming an ad-hoc network. Aggregator $(A)$ : aggregates information from all UAVs e.g. to produce a map showing the detected hazardous materials. Fig. 3 shows a simplified view of the self management framework using this example mission where a reconnaissance team comprised of the Commander, Surveyor and Relay roles is formed.

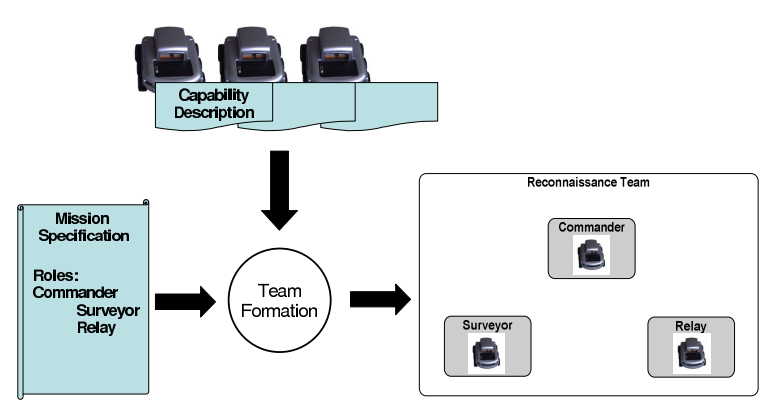

Fig. 3 Overview of the example mission

\subsection{Roles}

In this section we explain the concepts of a Role (R) as a placeholder containing specified role-missions (RM), authorisations (A) and tasks (T) which are loaded onto discovered UAVs that are assigned to the role. A role has an external (E) and a local (L) interface which provides a context for which role-mission policies can be specified. Fig. 4 shows the representation of a role and the components are explained below: 


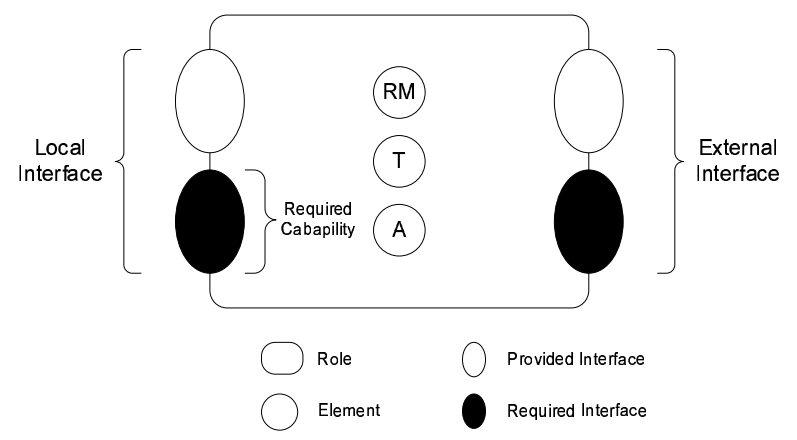

Fig. 4 Components of Role

Role Mission (RM): a set of policies relating to a single role for controlling tasks and enabling/disabling other policies. It is downloaded to a UAV assigned to the role.

Authorisation Policies (A): specify how roles are permitted to interact with each other in terms of the events that can be triggered or operations that can be invoked via the external interface.

Tasks (T): are complex operations which the UAV can perform e.g. move from $\mathrm{A}$ to $\mathrm{B}$, follow a path, track an object using video. Obligation policies in the mission may invoke operations supported by a task or activate a task. The tasks in a role are usually inherent to the type of the role and hence are specified inside the role class.

External Interface: defines operations and events relating to interaction with external collaborating roles: (i) Management Operations typically invoked by the commander for loading missions, policies etc. These are common to all roles. (ii) Provided Operations from the local interface, implemented by tasks in the role and hence are role specific. These are made visible to and can be invoked by other roles in remote UAVs. (iii) Required Operations that this role may invoke on other collaborating roles. (iv) Outgoing Events generated by the tasks inside the role or propagated from the UAV components such as sensors, and published via an event bus for use by other roles e.g. to trigger obligation policies. (v) Incoming Events generated by collaborating roles and required by this role, to trigger policies.

Local Interface: defines operations and events within the UAV for use by the role-mission executing on the UAV: (i) Operations implemented by the tasks within the UAV and invoked by local role-mission obligation policies. (ii) Events generated by the tasks within the UAV or propagated from UAV components such as sensors. These may trigger policies in the local role-mission or map to the external interface,

A self-managing team is specified in terms of roles to which UAVs are assigned and this is elaborated in Section 4. Adaptation of the team requires an approach to specification of roles that allows modification of their functional as well as non-functional behaviours. Fig. 5 shows an example role specification, 


\begin{tabular}{|ll|}
\hline Type & Surveyor \\
External interface & Provided $=\{$ start, stop, getPicture $\}$ \\
& Required $=\emptyset$ \\
Local interface & Provided $=\{$ start, stop, reassign, getPicture $\}$ \\
& Required $=$ motion, camera $\}$ \\
Tasks & \{explore, detectHazard $\}$ \\
Role mission & axplore a given area \\
& when a hazard is detected call the hazard unit $\}$ \\
Authorisations & allow the commander to get pictures $\}$ \\
\hline
\end{tabular}

Fig. 5 Example Role Specification

and high-level mission statements, from which a mission administrator can create a role.

\subsection{Mission}

A mission is a set of sequential or concurrent tasks which must be performed by multiple UAVs in order to achieve a goal. Since the context of a mission may change during execution, it is imperative that a mission specification for autonomous systems should allow adaptation of missions by enabling/disabling roles and changing UAV assignments to roles.

A team of UAVs should be able to perform a mission with a minimum number of UAVs that have the required capabilities although the configuration may not be optimal. When additional UAVs become available the team should expand to make use of the new resources, thereby increasing performance. Should there be a failure or departure of UAVs from the enlarged team, the team should contract but continue the mission. We define a minimal team configuration as the fewest types and number of UAVs needed to accomplish a mission. A mission can only be started when a team satisfying the minimal configuration can be formed, else it fails.

This concept is illustrated using the reconnaissance scenario in which the minimal configuration is defined to be one Commander, one Aggregator, and two Surveyors, where the Surveyor role is the primary role; and the Relay and Hazard detection roles are secondary roles. As shown in Fig. 6(a), the Surveyor role is collocated with the Relay and Hazardous material detector roles. The Surveyor role can be performed in parallel with either the Relay or the detector role while the UAV has to switch between the Relay and detector roles as only one of these can be active at a time. Although the Relay role can run in parallel with the Surveyor role, it will potentially hinder the surveying duty when trying to maximise communication link quality. Hence this role is best placed in another UAV should there be one available. Thus UAV to role assignment should be optimised to achieve a reasonably optimal configuration as explained in Section 4.

A reasonably-optimal mission configuration is defined to be one Commander, two Surveyors, two Hazardous material detectors, two Relays, and one 


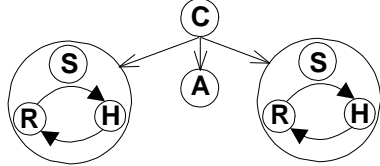

(a) Minimal

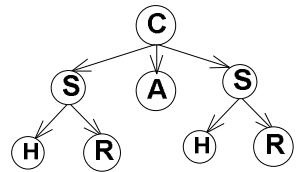

(b) Optimal

Fig. 6 Reconnaissance Mission Configuration

Aggregator each allocated to a separate UAV. The team started with the configuration shown in Fig. 6(a) and reached the configuration shown in Fig. 6(b) as new UAVs join the team in the mission area. The Surveyor roles which assigned the Relay and Hazard detector roles serve as managers for those two roles. Should any of the new UAVs fail or depart, the mission will revert to its minimal configuration.

A mission for a team of UAVs can be described in terms of roles, the management and reporting relationships between roles, constraints and mission parameters. We define the manages relation between two roles $\mathrm{x}$ and $\mathrm{y}$ as manages $(x, y)$ where role $\mathrm{x}$ is responsible for assigning a UAV to role $\mathrm{y}$ and withdrawing it. The reports relationship reports $(x, y)$ specifies role $\mathrm{x}$ is responsible for sending state information to role y periodically as specified by role y. We consider two types of constraints namely cardinality and collocation. The cardinality constraint sets the maximum number of roles a given type of role can manage and it is specified as a role-type and value pair. It also specifies the maximum number of roles that can be assigned to a single autonomous system as part of a single mission. The collocation constraint indicates the type of roles that cannot be placed together. Note that if two or more roles have to be collocated then they can be specified as tasks and placed in a single role. Using the aforementioned entities we define a mission, $M$, as follows ( $V$ is a set containing all the mission roles and $E$ is a management relation between the roles, while $P$ and $C$ are sets of mission parameters and constraints respectively):

$$
\begin{aligned}
& M=<V, E, P, C>\quad \text { where } \\
& V=\left\{R_{1}, R_{2}, R_{3}, \ldots R_{i}\right\} \\
& E=\{(x, y) \mid x \in V \wedge y \in V \wedge x \neq y \wedge \operatorname{manages}(x, y) \wedge \text { reports }(y, x)\} \\
& P=\left\{P_{1}, P_{2}, P_{3}, \ldots P_{j}\right\} \\
& C=\left\{C_{1}, C_{2}, C_{3}, \ldots C_{k}\right\}
\end{aligned}
$$

In order to allow adaptation and reuse we specify missions in three levels namely policy, mission class and mission instance as shown in Fig. 7. Policies are specified using Ponder2 [35] and stored in a policy repository. A mission class is an XML specification of constraints, mission parameters, types of roles needed for the mission and the management relation among the roles while a mission instance is an XML specification that defines the mission parameters and role cardinalities required to instantiate a mission class. A policy from 
the repository can be used by multiple mission classes and there can be multiple mission instances instantiated with different parameters from a particular mission class as shown in Fig. 7. The policy repository is comparatively small so can be stored in the commander's memory and policies may be distributed to other UAVs as needed or fetched by corresponding roles directly.

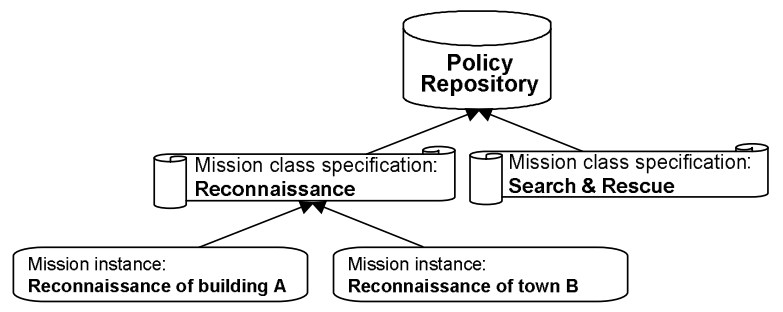

Fig. 7 Mission Specification Levels

\subsubsection{Policy Specification}

As mentioned earlier, policies are specified using Ponder2 [35] because of its support for both explicit specification of authorisation and obligation policies which are necessary for our framework, its extensibility, and its scalability in that its interpreter is scalable enough to be deployed on systems with constrained resources. Ponder 2 is a generic object management system which allows dynamically loading, unloading, enabling and disabling of managed objects. Generally a Ponder 2 managed object is an active object that is capable of receiving action commands and performing actions. A managed object can be either part of the management system's architecture such as a policy or an adaptor to a managed sensor. Managed objects are written in Java. PonderTalk is a Smalltalk-like language used for configuration and control of a Ponder2 system by specifying commands for controlling and interacting with managed objects via policies. A PonderTalk specification is a set of statements separated by a "." that are either assignments (to a temporary variable created on the fly) or message interactions which reference a managed object followed by zero or more commands (messages) sent to the managed object. Message interactions have return values, which are PonderTalk objects, and hence they can be assigned to variables or passed as data for other commands.

Events and policies are specified using PonderTalk and their creation is facilitated by event and policy factory objects namely EventTemplate, ObligationPolicy and AuthorisationPolicy, which are provided by Ponder2. These factories are created and stored in the domain structure as shown in Fig. 8.

Ponder 2 events are a set of name-vale pairs with a name list defined in an event template. Each event has an event template (type) which is created by sending a create message with the list of argument names to the event template factory as shown in Fig. 9. 


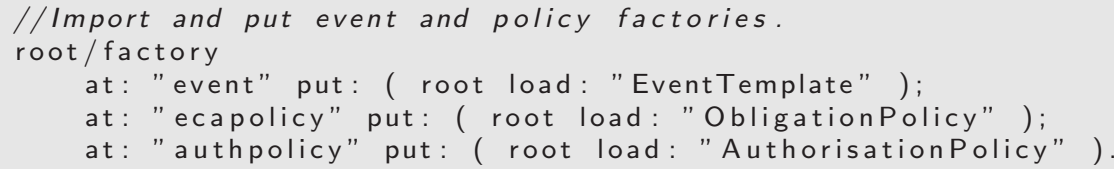

Fig. 8 Ponder2 event and policy templates

event-factory create: arguments-array

Fig. 9 Syntax of a Ponder2 event type

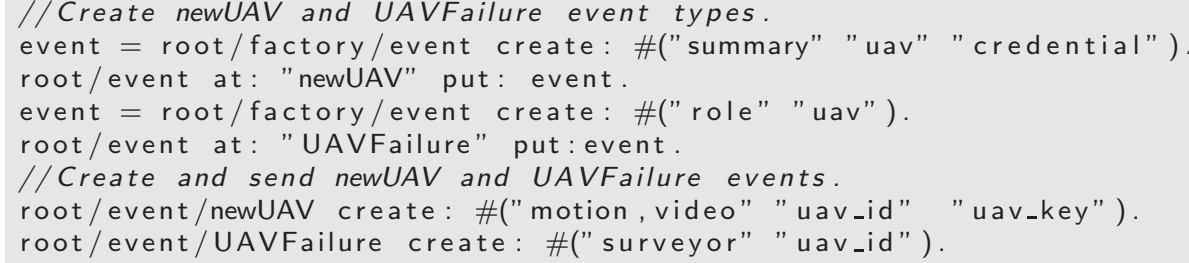

Fig. 10 Example Ponder2 events

policy $:=$ root/factory/ecapolicy create.

policy event: event-type;

condition : [: arg|boolean-expression];

action : [: arg|statements]

Fig. 11 Syntax of a Ponder2 obligation policy

Once the event template is created, an event of that type can be created by sending messages, with or without values, to the template. After the event is created it is propagated to all policies that have subscribed to that event type. Fig. 10 shows example Ponder2 event types and events. Events can also be created by managed objects (i.e. in the Java code of the managed object) provided that they are given the event template for the events they are expected to generate and they understand the semantics of the event types (i.e. the arguments). The event templates can be passed to the managed objects through messages.

Obligation policies are created by using the obligation policy factory object and setting the event type, condition and actions of the policy. The event type, condition and actions are set through a message sent to the policy object. The condition and actions are specified using blocks as shown in Fig. 11. When an event of the specified type occurs, the policy evaluates the condition block and if the result is true, it executes the action block. The blocks get the values for their arguments from the event.

Authorisation policies are created using the authorisation policy factory object and specifying the subject, action, target and focus of the policy, as shown in Fig. 12. The focus indicates whether the authorisation policy protects 
policy $:=($ root $/$ factory/authpolicy

subject : subject-path-and-name

action : action-name

target : target-path-and-name

focus : focus-type).

Fig. 12 Syntax of a Ponder2 authorisation policy

the source (subject), target or both from a given action. The source can be protected from performing actions or accepting replies to actions that are harmful to itself or other subjects in the domain. The target can be protected from unauthorised subjects trying to perform an action (access control) or from sending back the result of an action that contains sensitive information (privacy control).

The policies specified for a role are broadly divided into role assignment policies, used to assign UAVs to roles based on their capabilities and operational management policies used by roles to manage their own or collaborating roles' operational behaviour. In the example role assignment policy shown in Fig. 13(a), the commander checks the capability of a newly discovered UAV, authenticates it and assigns it to the surveyor role, if it has the required capability. Fig. 13(b) shows an example re-assignment policy to deal with a failure.

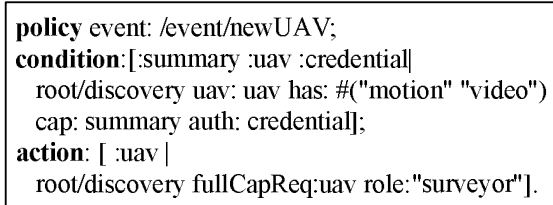

(a) Initial Role Assignment

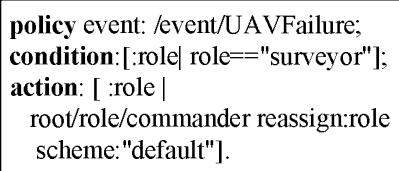

(b) Role Re-Assignment

Fig. 13 Sample Ponder2 Policies

\subsubsection{Mission Class and Mission Instance Specifications}

A mission class specification specifies a team in terms of roles, policies a role uses to manage itself or other roles (where hierarchy exists) and indicates the management relation among the participating roles as well as the cardinality of each role. Mission parameters such as failure-timeout which are shared by all roles are also included. This specification can be used to instantiate different teams of the same configuration with different cardinalities, mission parameters and role behaviours using policies. The policy-based role behaviour specification allows for changing the behaviours of assigned roles.

Fig. 14(a) shows the mission specification for the reconnaissance scenario with a commander role managing a surveyor and an aggregator role. The surveyor role in-turn manages the hazard-detector and relay roles. A mission 


\begin{tabular}{|c|}
\hline 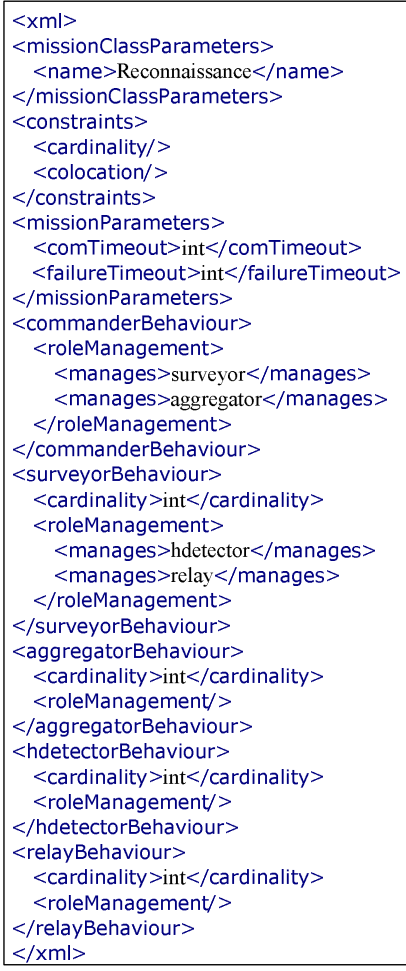 \\
\hline
\end{tabular}

(a) Mission Class Specification

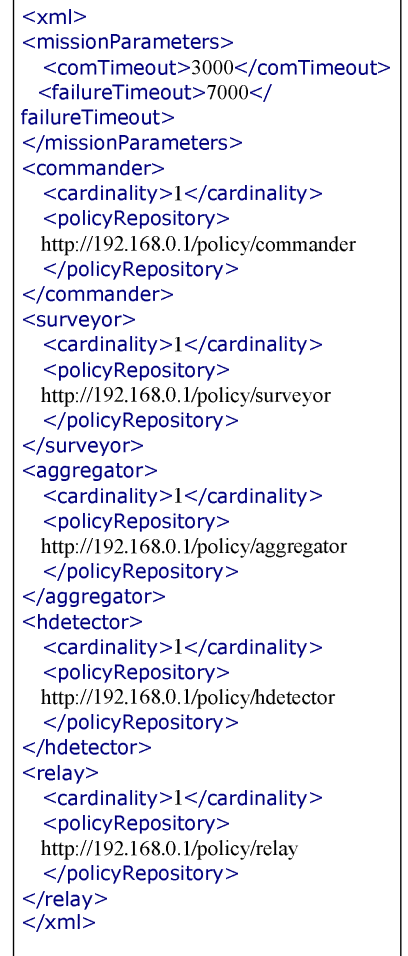

(b) Mission Instance Specification

Fig. 14 Mission Specification

instance (which gives rise to the actual team of UAVs performing the mission) specifies values for cardinalities, mission parameters and URIs of policies which define the role behaviour as shown in Fig. 14(b).

\section{Team Management}

A commanding officer specifies a mission involving a team of UAVs which need to collaborate to achieve some overall goal such as locating hazardous material or a search and rescue. Although there is initially a single specification within the mission commander, it is distributed to UAVs as they are discovered and assigned to roles within the mission, according to their capabilities. The new team members can also perform team management functions of discovering other UAVs and assigning them to roles, as defined by the mission specification. As UAVs are added to the team they form a hierarchical tree management structure in that a node effectively manages the UAVs it discovers. Status report go up the hierarchy. The hierarchy forms a distributed self-managing mission. The mission specification defines the hierarchical structure amongst 
roles. However, the actual hierarchical structure amongst UAVs is not predefined as it will depend on the order in which UAVs are discovered and their capabilities which define which roles they are assigned to.

A team of UAVs can be described in terms of roles the UAVs are assigned to and the relationship among these roles, and can be modelled as a digraph with the roles as vertices and their relationship as edges. Using the manages and reports relationships defined in Section 3 we define a team, $\Gamma$, as follows:

$$
\begin{aligned}
& \Gamma=(V, E) \quad \text { where } \\
& V=\left\{R_{1}, R_{2}, R_{3}, \ldots R_{i}\right\} \\
& E=\{(x, y) \mid x \in V \wedge y \in V \wedge x \neq y \wedge \text { manages }(x, y) \wedge \operatorname{reports}(y, x)\}
\end{aligned}
$$

\subsection{Team Formation}

The team layer of the management framework deals with the formation of a distributed self managing team of UAVs by discovering new UAVs within the communication range of those which are already part of the team, authenticating them [14], assigning them to suitable roles based on their capabilities, and maintaining the team. The team formation process is facilitated by five interacting services residing in the team layer. These are the Capability, Role manager, Discovery, Assignment services and the Role itself that is being enacted by the UAV. The role manager deals with management tasks that are common for all roles such as loading, starting and stopping a role as well as providing support for running multiple roles on a single UAV.

\subsubsection{Discovery}

A manager role runs a discovery service to discover new UAVs and assigns them to one if its managed roles. When a new UAV comes into the communication range of the manager UAV it will be able to receive the broadcast. The new UAV then replies to the broadcast with a join request if it is willing to take part in a mission. This leads to the initiation of a mutual authentication protocol which, if it succeeds, results in a shared secret key [14]. Using this key, the discovered UAV will encrypt its capability summary and send it to the manager UAV. Encrypting the capability summary is necessary to prevent disclosure of a UAV's services to a third party as this may be sensitive information in military missions. Upon receiving the capability summary the manager $\mathrm{UAV}$, using its role assignment policies, decides whether the discovered UAV has the potential to be assigned to one of the roles and if so it will request the discovered UAV for its full capability description. If the manager UAV is satisfied with the full capability description it will assign the discovered UAV to an appropriate role. The newly assigned UAV might run its own discovery service if it has roles to be assigned, and assigned UAVs stop responding to discovery broadcasts. 
The capabilities of a UAV indicate its ability and potential in terms of the basic resources and functionalities that are expected by the tasks and policies associated with the role that is to be assigned to the UAV. The assignment of a UAV to a role is based on matching offered capabilities from a discovered UAV, with the required capabilities defined in the role definition.

When devising a scheme for describing capabilities, the two major issues to be addressed are identifying the type of information to be provided by a capability description and specifying a representation language for the description. Based on the type of information they carry, we have identified two levels of UAV capability descriptions namely, capability summary and full capability description. A capability summary shows the low-level resources of the UAV and a summary of services provided by the UAV. For example if we say that a UAV has a video camera, a long range communication link, and a map builder service we are describing the UAV's hardware resources as well as services although the description does not give information on how to use them. The rationale for using this summary as a capability description is to provide information about the potential of the UAV which influences the decision to which roles it can be assigned.

A full capability description defines the types of services a UAV provides and the list of associated operations and notifications relating to the use of the service. For example a UAV which is capable of streaming a video might include in its service description that it can stream video, and supports control actions such as start, stop, pan camera etc. UAVs provide different kinds of services such as mine detection, video streaming etc. Roles assigned to these UAVs may need to use these services (tasks) as stand alone services or they may combine different services and create a new functionality.

We found the UPnP [41] approach to service description to be the most fitting to our framework since it can describe both the capability summary and full capability. Our capability description has three main parts, a system section that provides general information such as the name, owner, manufacturer, model etc. of the UAV as well as credentials and contextual information such as battery power and location, a device list section that provides a list of the devices embedded in the UAV such as processors, sensors etc., and a service list section which describes the list of services provided by the UAV. Unlike the UPnP service description, we specify the directions of all operations. This enables us to explicitly state what operations a service uses and what operations it provides, thus enabling dynamic service composition.

In parallel with our two levels of capability description, we have two levels of capability matching. The first one deals with matching the role's required capabilities (as specified in the local interface of the role) to the capability summary of the UAV in order to determine whether the UAV can accommodate the role or not. The result of this matching is: (i) Match: if the UAV has all the devices and services required by the role or it is a superset and some more additional capabilities, (ii) No match: if the UAV does not have all of 
the devices and services required by the role. Note that a UAV that has only a subset of the required capabilities will be categorised as no match.

The second level deals with matching the quality of service required by the role, and the tasks of the role with the full capability description of the UAV. The result of the quality of service matching may be used to adapt the behaviour of the role to the UAV's capability or it may lead to rejection of the UAV should there be a policy enforcing a minimum level of quality of service and the UAV's capability does not meet that. Both the capability summary and full capability matching assume an agreed ontology between the discovering UAV and the discovered UAV.

\subsection{Role Assignment}

A UAV is assigned to one or several of the roles in the mission based on its capabilities which it provides to the managing role during discovery. This decision can be made either immediately or in a delayed manner giving rise to an immediate or optimised role assignment respectively as described below.

\subsubsection{Optimal Role Assignment}

An immediate role assignment assigns a UAV to an available role, immediately on discovery, without considering other roles and possible future discoveries. Consider a reconnaissance team with roles Commander (C), Surveyor (S), Aggregator (A), Relay (R), Hazard-detector (H), with their capability requirements shown in Fig. 15(a).

\begin{tabular}{ll}
\hline Role Type & Required \\
\hline Surveyor $(\mathrm{S})$ & $\{$ video, motion $\}$ \\
\hline Aggregator $(\mathrm{A})$ & $\{$ map $\}$ \\
\hline Relay $(\mathrm{R})$ & $\{$ motion, lrangecom $\}$ \\
\hline Hazard Detect $(\mathrm{H})$ & $\{$ motion, hdetection $\}$
\end{tabular}

(a) Capability requirement of roles

\begin{tabular}{ll}
\hline UAV & Provided \\
\hline$U A V_{1}$ & $\{$ map, video, motion $\}$ \\
\hline$U A V_{2}$ & $\{$ map, video, motion, lrangecom $\}$ \\
\hline$U A V_{3}$ & $\{$ video, motion $\}$ \\
\hline$U A V_{4}$ & $\{$ motion, hdetection $\}$ \\
\hline
\end{tabular}

(b) UAV Capabilities

Fig. 15 Capabilities

Assuming that the Commander role is already assigned at time $t_{0}$ this mission needs four UAVs. Now let us assume that four UAVs, with capabilities shown in Fig. 15(b), come within the Commander's communication range as indicated by the arrival order shown in Fig. 16. $U A V_{1}$ is discovered at time 


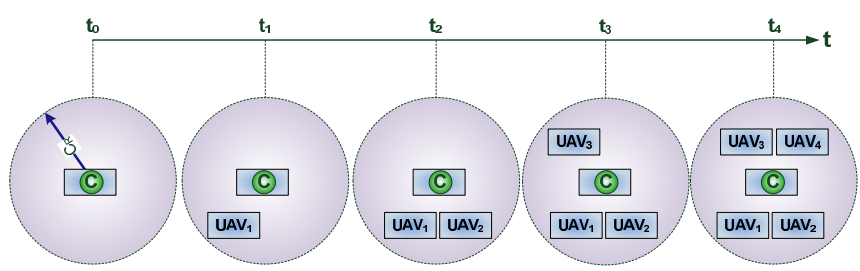

Fig. 16 UAV Arrivals

$t_{1}$ and the Commander's role assignment policies, shown in Fig. 17(a), dictate that it be assigned to either a Surveyor or an Aggregator role. Although $U A V_{1}$ has more capabilities than required by a Surveyor role, since it satisfies the capability requirement of both roles it can be assigned to either one of them. Assuming the worst case scenario let us say that it is assigned to the Surveyor role. $U A V_{2}$ is discovered at time $t_{2}$ and it will be assigned to the remaining role, i.e., the Aggregator. $U A V_{3}$ and $U A V_{4}$ are discovered at times $t_{3}$ and $t_{4}$ respectively by the Aggregator which is now running a discovery service since it needs to assign two roles. However, $U A V_{3}$ does not satisfy the capability requirement of either one of them and hence it will not participate in this mission, $U A V_{4}$ will be assigned to the Hazard-detector role. The Relay role will never be assigned leaving the mission in an incomplete team configuration until (if at all) a UAV with the necessary capabilities is discovered.

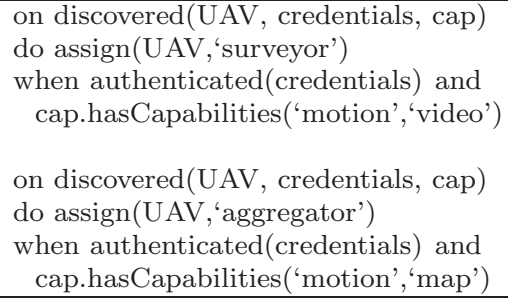

on discovered(UAV, credentials, cap)

do assign(UAV,'surveyor')

when authenticated(credentials) and

cap.hasCapabilities('motion', 'video')

on discovered(UAV, credentials, cap)

do assign(UAV,'aggregator')

when authenticated(credentials) and cap.hasCapabilities ('motion','map')

(a) Commander

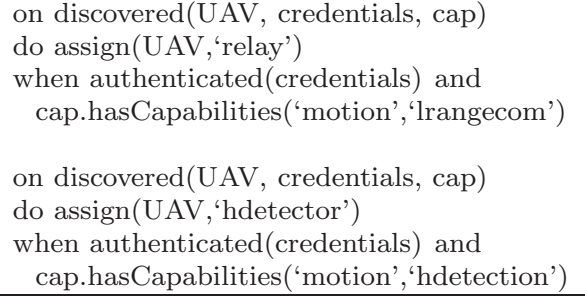

(b) Aggregator

Fig. 17 Role Assignment Policies

Consider two UAV arrival orders different from the one shown in Fig. 16, $U A V_{1} \rightarrow U A V_{3} \rightarrow U A V_{2} \rightarrow U A V_{4}$ and $U A V_{2} \rightarrow U A V_{1} \rightarrow U A V_{3} \rightarrow U A V_{4}$ where $a \rightarrow b$ stands for $U A V$ a is discovered before $U A V b$. Using the role assignment policies of the Commander and the Aggregator, shown in Fig. 17, in the worst case scenario we may end up with assignments $\left\{S \mapsto U A V_{1}\right.$, $\left.A \mapsto U A V_{2}, H \mapsto U A V_{4}, R \mapsto \emptyset\right\}$ and $\left\{S \mapsto U A V_{2}, A \mapsto U A V_{1}, H \mapsto U A V_{4}\right.$, $R \mapsto \emptyset\}$ respectively where $r \mapsto s$ stands for role $r$ is assigned to $U A V s$. In both cases we end up with an incomplete team configuration.

In the first case the problem was initially caused due to the fact that when deciding to assign the Surveyor role to $U A V_{1}$ the Commander did not take the other role (Aggregator) waiting to be assigned, into consideration leading 
to a later assignment of this role to a UAV which has a scarce capability, long range communication, required by the relay role. This problem could have been avoided if the Commander had taken all its roles waiting to be assigned into consideration, instead of immediately assigning discovered UAVs.

In the second case, the problem was caused by the Commander again using a UAV with a scarce capability that is needed by the relay role. However, this problem could not have been avoided even if the Commander had taken all its waiting roles into consideration since both $U A V_{2}$ and $U A V_{1}$ satisfy the capability requirement of the Surveyor and Aggregator roles.

From the above scenarios, we can observe that immediate role assignment leads to incomplete team configurations because of the lack of local (the Commander not considering the Aggregator) and global (the Commander not considering the Relay) consideration of future role assignments.

The team formation starts from the UAV which is initially loaded with the mission specification, this UAV assigns roles managed by it and these roles in turn repeat this process until the team reaches its final configuration. If the roles take local and global future assignments into consideration the team will reach its optimal configuration provided that UAVs with the necessary capabilities are available. To attain this we define two objectives a manager role should achieve: (i) Local objective: a manager role should assign all its managed roles to the best available UAVs. (ii) Global objective: a manager role should facilitate the assignment of all roles managed by its managed roles. Given a set of roles and UAVs there will be a number of permutations of assignments. In order to compare and choose from these possible assignments, a means for evaluating the desirability of each assignment is necessary. Utility functions which ascribe real-valued desirability to each assignment can do that. Utility functions enable a fine grained and flexible way of specifying a desirable behaviour in contrast to a more strict approach that classifies a behaviour as desirable and undesirable [23]. We model the role assignment problem using roles, UAVs, the management relation amongst roles, constraints and utilities as shown in Fig. 18. The functions $r e q_{c a p}$ and prov $_{c a p}$ express the required and provided capabilities by roles and UAVs respectively. battery $(s)$ gives the available battery power of a UAV. Management relations are expressed through the managed by (represented as mgdby) and management closure (represented as mgmnt $_{\text {closure }}$ ) relations. The $m g d b y$ relation defines the set of roles managed by this role, while the management closure of a role is all the roles managed by it directly and indirectly. $u(r)$ gives a measure of the benefit acquired by using a role $r$, for example, the utility can correspond to the priority of a role. $u(s)$ gives a measure of the benefit acquired by using UAV $s$, for example, more battery power corresponds to a higher utility level. $u(r, s)$ gives a measure of the benefit acquired by assigning role $r$ to UAV $s$. For example, if a manager role is concerned with global objectives the utility of an assignment of a role to a UAV which has the exact capability requirements is higher than the utility when using a UAV with more capabilities than required by the role. On the other hand, if a role is concerned only with local objectives the utility may be the same for all assignments that satisfy the capability 
1. A set $R$ of $n$ roles with their attributes

$$
\begin{aligned}
& R=\left\{r_{1}, r_{2}, r_{3}, \ldots, r_{n}\right\} \\
& A \text { set } \quad \text { of } \quad t \text { capabilities. } \\
& C=\left\{c_{1}, c_{2}, c_{3}, \ldots, c_{t}\right\} \\
& \text { req } q_{\text {cap }}: R \rightarrow \mathcal{P}(C) \text { where } \mathcal{P}(C) \text { is the power set of } C
\end{aligned}
$$

2. A set $\mathrm{S}$ of $k \mathrm{UAVs}$ with their attributes

$$
\begin{aligned}
& S=\left\{s_{1}, s_{2}, s_{3}, \ldots, s_{k}\right\} \\
& \operatorname{prov}_{\text {cap }}: S \rightarrow \mathcal{P}(C) \\
& \operatorname{battery}_{(s): S \rightarrow \Re^{+}} \\
& \operatorname{cred}(s)=\text { credentials of a UAV }
\end{aligned}
$$

3. A management relation amongst roles

$$
\text { mgdby }: R \rightarrow \mathcal{P}(R) \quad \text { where }
$$$$
r_{i} \in \operatorname{mgdby}\left(r_{j}\right) \quad \text { if } \quad r_{j} \text { is responsible for assigning } r_{i}
$$
management closure $_{\text {: }} R \rightarrow \mathcal{P}(R)$

$$
\text { mgmnt }_{\text {closure }}\left(r_{j}\right)= \begin{cases}r_{j} & \text { if } \\ \bigcup_{r_{i} \in m g b d y\left(r_{j}\right)} m g \operatorname{mgdy} t_{\text {closure }}\left(r_{i}\right)=\emptyset & \text { otherwise }\end{cases}
$$

4. A set of collocation constraints that must be satisfied by a valid role assignment.

$$
\begin{aligned}
& \text { colloc : } R \times R \rightarrow\{-1,0,1\} \\
& \text { colloc }\left(r_{i}, r_{j}\right)= \begin{cases}-1 & \text { if } r_{i} \text { and } r_{j} \text { cannot be assigned to the same UAV } \\
0 & \text { if there is no restriction } \\
1 & \text { if } r_{i} \text { and } r_{j} \text { have to be assigned to the same UAV }\end{cases}
\end{aligned}
$$

5. A set of utility functions.

$$
\begin{aligned}
& u(r): R \rightarrow \Re^{+} \\
& u(s): S \rightarrow \Re^{+} \\
& u(r, s): R \times S \rightarrow \Re^{+} \\
& w(u(r)): U_{\text {role }} \rightarrow \Re^{+} \\
& w(u(s)): U_{U A V} \rightarrow \Re^{+} \\
& w(u(r, s)): U_{\text {role }, U A V} \rightarrow \Re^{+} \\
& U_{\text {role }}=\left\{u_{1}(r), u_{2}(r), u_{3}(r) \ldots u_{m}(r)\right\} \\
& U_{U A V}=\left\{u_{1}(s), u_{2}(s), u_{3}(s) \ldots u_{p}(s)\right\} \\
& U_{\text {role }, U A V}=\left\{u_{1}(r, s), u_{2}(r, s), u_{3}(r, s) \ldots u_{q}(r, s)\right\}
\end{aligned}
$$

Fig. 18 The Role Assignment Model 


$$
\begin{aligned}
& \text { Find a function } f: R \rightarrow S \text { that maximises } U \text { without violating the constraints } \\
& \begin{array}{l}
U=\sum_{j=1}^{n} \sum_{l=1}^{k} U_{j, l} \\
U_{j, l}=\sum_{i=1}^{m} u_{i}\left(r_{j}\right) * w\left(u_{i}\right)+\sum_{i=1}^{p} u_{i}\left(s_{l}\right) * w\left(u_{i}\right)+\sum_{i=1}^{q} u_{i}\left(r_{j}, s_{l}\right) * w\left(u_{i}\right)
\end{array}
\end{aligned}
$$

Fig. 19 The Role Assignment Problem

matching requirements. $w(u)$ provides the weight associated to each utility function. For example in one mission scenario, a utility measuring the battery power may be given more weight than a utility that measures the degree of capability matching. However, this choice may change in another scenario and hence the need to model utility weights as functions.

Using the role assignment model shown in Fig. 18 we formulate the role assignment problem as shown in Fig. 19. To solve this problem we model it as a weighted bipartite-graph minimum-cost maximum-matching problem where one partition of vertices are the roles and the other partition of vertices are the UAVs. The weights of the edges in the graph are computed using the utility (treating maximum utility as minimum cost) shown in Fig. 19.

Efficient algorithms, such as Hopcroft and Karp's $O(m \sqrt{n})$ algorithm [15], where $m=n d, n=$ number of vertices and $d=$ degree of each vertex, exist for solving the maximum matching problem for regular bipartite graphs, which would satisfy our local objective. However, to satisfy both the local and global objectives, i.e., in order not to use UAVs which have more capabilities than required by any of the roles that are going to be assigned by the current manager role, we are interested in minimum cost in addition to maximum matching. Algorithms, such as the polynomial time Hungarian algorithm $[27,26]$, that can compute the minimum-cost maximum matching for regular bipartite graphs exist. We can easily transform the role assignment bipartite graph to a regular graph by adding dummy roles or UAVs if we have more UAVs than roles or vice versa respectively. The collocation constraint $\operatorname{colloc}\left(r_{i}, r_{j}\right)=-1$ is satisfied by default because the bipartite matching model assigns each role to a unique UAV. We do not consider the $\operatorname{colloc}\left(r_{i}, r_{j}\right)=1$ constraint because, although collocation of roles is supported to enable resource (UAV) sharing in the face of scarcity, roles should not be forced to be collocated as those that must be placed together can be designed as tasks and placed in one role. However, should there be a need to consider it, our problem solving model is capable of dealing with this constraint which can be easily encoded into the matching problem by defining a third role $r_{k}$ where $r e q_{c a p}\left(r_{k}\right)=r e q_{c a p}\left(r_{i}\right) \cup r e q_{c a p}\left(r_{j}\right)$.

We specify the weight function, $w(u)$ for utilities using policies in order to make the optimisation system flexible and adaptable. The role assignment algorithm is shown in Algorithm $1, R_{w}$ is the set of roles waiting to be assigned and $T_{w}$ is the waiting period before optimisation is started. 


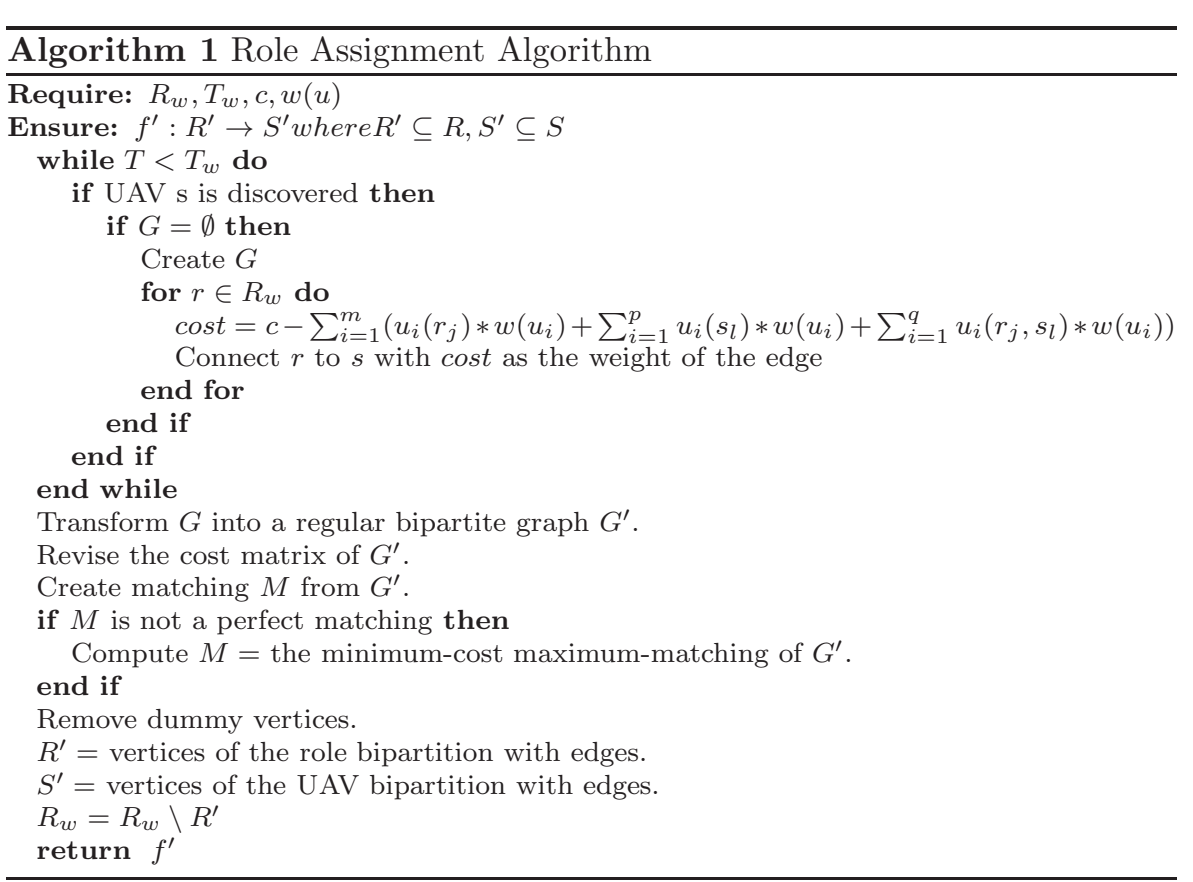

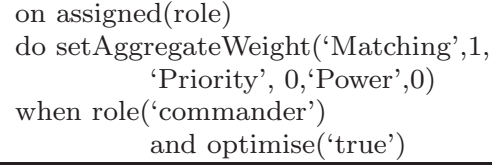

(a) Weight Policy

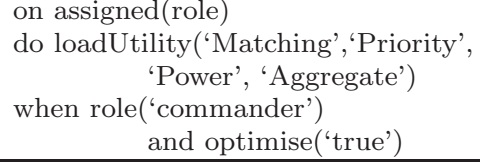

when role('commander') and optimise('true')

(b) Utility Loading Policy

Fig. 20 Example Policies

\subsubsection{Utility Models for Optimal Role Assignments}

We use three utility functions for optimal role assignments, a role utility, a UAV utility and role-UAV utility. The role utility, priority $(r)$, measures the benefit of assigning role $r$ with respect to the priority this role has compared to other roles. The UAV utility, power $(s)$, measures the benefit of using UAV $s$ with respect to battery power compared to other UAVs. The matching $(r, s)$ utility measures the benefit of assigning role $r$ to UAV $s$ with respect to capability matching. The overall utility is computed by the AggregateUtility which uses the weights provided through policies (Fig. 20(a)) for each utility. AggregateUtility checks all utilities which are loaded into the management system (Fig. 20(b)), identifies their types and aggregates their values, thereby enabling the addition of new utility functions dynamically using policies. The utility functions and their corresponding weights are specified by the mission administrator. Although the utility function based approach is powerful in 


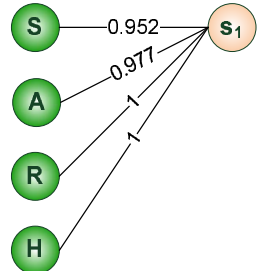

(a)

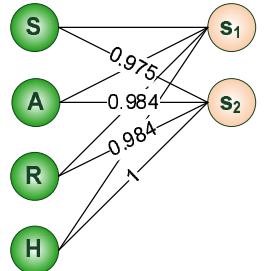

(b)

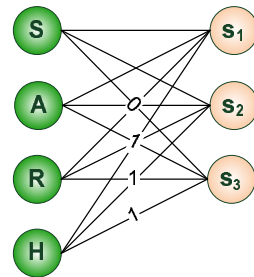

(c)

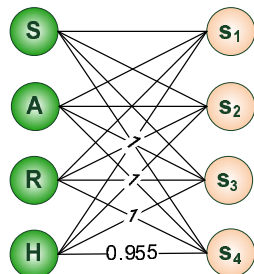

(d)

\begin{tabular}{|l|l|l|l|r|}
\hline & \multicolumn{1}{|c|}{$\mathbf{S}_{\mathbf{1}}$} & \multicolumn{1}{c|}{$\mathbf{S}_{\mathbf{2}}$} & \multicolumn{1}{c|}{$\mathbf{S}_{\mathbf{3}}$} & \multicolumn{1}{c|}{$\mathbf{S}_{\mathbf{4}}$} \\
\hline $\mathbf{S}$ & 0.952 & 0.975 & 0 & 1 \\
\hline $\mathbf{A}$ & 0 & 0.007 & 0.023 & 0.023 \\
\hline $\mathbf{R}$ & 0.016 & 0 & 0.016 & 0.016 \\
\hline $\mathbf{H}$ & 0.045 & 0.045 & 0.045 & 0 \\
\hline
\end{tabular}

(e)

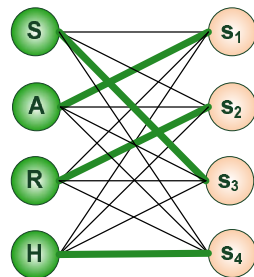

(f)

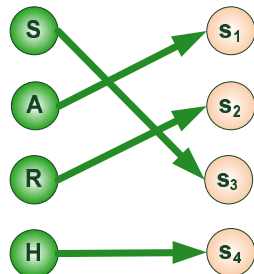

(g)

Fig. 21 Trace of the Assignment Algorithm

on discoveryReady()

do setOptimRate('1000')

Fig. 22 Optimisation Rate Policy

many ways, designing the function and deciding the weights are challenging tasks.

Consider the reconnaissance team with the required and provided capabilities shown in Fig. 15 and with the UAV arrival order, $U A V_{1} \rightarrow U A V_{2} \rightarrow$ $U A V_{3} \rightarrow U A V_{4}$, as shown in Fig. 16. We have previously shown that the team will never become complete with this arrival order, we will now show that using the optimal assignment algorithm the team will reach its optimal configuration.

Fig. 21 illustrates traces of the optimal assignment algorithm execution with the inputs of the algorithm $T_{w}=$ waiting time before optimisation= $1000 \mathrm{~ms}, c=$ constant to change utility into cost $=1, w(u)=$ weight of a utility function, set by the policies shown in Figures 20(a) \& 22. As it can be seen from the policy in Fig. 20(a), for this example, the power and priority utilities are not included in the aggregate utility computation because their weight is set to zero which makes the matching utility the deciding factor for assignment.

The utility function used for matching is $u(x)=\frac{1}{a *(1+x)}$ where $x=r \triangle s$, is the symmetric difference between role $r$ 's required capability and UAV $s$ 's provided capability. We use Bloom filters [6] to represent the capability descriptions, this enables us to compute set operations efficiently. 
A matrix representation of the role assignment graph is created dynamically when UAVs are discovered. As shown in Fig. 21(a), when $s_{1}$ is discovered its matching utility is computed with respect to each role waiting to be assigned. The same applies for UAVs $s_{2}, s_{3}$ and $s_{4}$, by which time the timer set by the policy shown in Fig. 22 goes off and the optimisation starts. The input to the optimiser is the matrix representation of the role assignment bipartite graph. In our example this matrix corresponds to Fig. 21(d) with the weights for each edge computed in the steps shown in Fig. 21(a) - (d). The output is a cost matrix representing the minimum-cost maximum matching of the graph that is computed by using the Hungarian algorithm. The Hungarian algorithm starts by revising the input cost matrix (for each row and column subtracting the minimum entry in the row and column from all the elements corresponding to that row and column respectively) and then iteratively searches for an assignment that results in the maximum number of nodes on one side of the bipartite graph being assigned to the other. The resulting cost matrix ${ }^{1}$ of the optimisation, for the example assignment, is shown in Fig. 21(e) and the edges of the bipartite graph which have zero cost are marked as shown in Fig. 21(f). The assignment will then take the edges with the lowest cost for each role as shown in Fig. 21(g) resulting in an optimal configuration where every role is assigned to the best possible UAV.

\subsubsection{Implementation}

The management architecture [13] has three layers, namely, mission, team and communication, as shown in Fig. 1. The optimisation task is performed in the team layer which consists of the Discovery and Optimiser entities. The Discovery entity periodically broadcasts discovery beacons over UDP, however subsequent communication between this entity and the discovered UAV uses the secure communication channel implemented by the Communication Handler entity in the communication layer. It interacts with the Optimiser entity to add a successfully discovered UAV to the optimisation process. The broadcast rate and other behaviours of the discovery service can be adapted through policies.

The Optimiser entity receives the list of roles waiting to be assigned, from the role enacted by the local UAV and newly discovered UAVs from the discovery service. It builds a bipartite graph where the roles are one bipartition and the UAVs are the other. The graph building process continues until the timeout before optimisation (set by a policy) is reached. Our implementation of Bloom filters is done using the lower bound for false positives and the optimal value for the number of hash functions as described in [8]. The parameters of the Bloom filter (including the maximum allowable false positive) can be changed using policies.

When the timeout (which can be specified in the mission specification) before optimisation expires, the Optimiser computes a minimum-cost maxi-

${ }^{1}$ In this example, the optimal assignment is found right after revising the cost matrix. 
mum matching of the bipartite graph, using a variant of the $O\left(n^{3}\right)$ improved Hungarian algorithm $[27,26,33,7,10]$, and provides the result to the manager role which uses it to perform the role assignments. The management task is distributed as all roles can perform role assignments.

\subsection{Team Maintenance}

To ensure that the UAVs comprising the team perform their tasks correctly, it is important to cope with different types of failures. Consider the reconnaissance mission scenario. Failures in such missions can occur as a result of intermittent or permanent communication link failures as well as individual node failure. A recent study on UAV failures shows that reliability in field environments is only between 6 and 20 hours [9].

Our failure management scheme distinguishes between intermittent communication link disconnection and permanent UAV failures or isolation due to communication link failure. This scheme uses the management tree to define management hierarchies as well as data aggregation hierarchies during execution of the mission. If the periodical state information is not received within a specified timeout, it is considered that a failure has occurred. The timeouts are used to differentiate between the two types of failures and each failure is handled accordingly. An intermittent communication link disconnection does not require reassignment of roles as we assume that the UAVs will continue functioning in their normal roles but just not be able to communicate. However there may be some reconfiguration of the management hierarchy in that a subtree manager acts as commander of the disconnected subtree and, while disconnected, cannot report to its commander. The result, being, that the mapping of existing UAVs to roles remains the same whereas the management tree may be different, as it is assumed that the adaptation is temporary.

A permanent failure is caused by either a node or communication link failure (other UAVs cannot distinguish between these). The result is the partitioning of the team as well as a loss of roles. The partitioning problem is addressed using the approach mentioned earlier. The response to the loss of roles is as follows (in order of priority): (i) use replicated roles, if available, (ii) if there are unassigned or newly discovered UAVs, perform a role reassignment, while keeping the existing team configuration, to replace the lost role(s), and (iii) if none of the above is feasible, reconfigure the team by swapping less crucial roles for more crucial roles. If reconfiguration incurs role replacement, this takes place only in subsets of the team which are lower in the hierarchy than the failed UAV. This is due to the fact that roles assigned to higher level UAVs are assumed to be more crucial to the mission. In the case of role reassignment and reconfiguration, state information migration takes place.

While the failure management scheme copes with communication link failure and UAV failure, it is sometimes desirable to make sure that the members of the team modify behaviour in order to maintain communication links with the other members of the team. For this purpose, we propose the commu- 
nication management scheme. Previous works $([1,5,39,29,38])$ ensure this by making sure that the team of robots either restrict their motion to ensure that they do not loose communication or follow each other through line of sight. In our scheme, we use two approaches. In the first approach, we use a similar technique to the related works mentioned above by restricting the motion of some of the UAVs to ensure that they do not loose communication. While this scheme does alleviate the problem arising due to communication failure, it is also very restrictive with respect to the motion of the UAV. Consider the reconnaissance scenario where the UAVs would need to scope out an area as part of the mission. Restricting their motion will result in either the entire area not being explored or a substantial increase in the amount of time taken to explore that area as part of the mission. In the second approach, instead of restricting the motion of the UAVs, we use the concept of rendezvous area, which is an area where the UAVs belonging to the mission can gather at a specified time so that they can exchange the requisite information. In the event that a UAV is unable to reach the rendezvous area, it is assumed to have failed and the appropriate failure management mechanism is used. More details on the team maintenance schemes can be found in [14].

\section{Experiments and Results}

The prototype implementation was evaluated through experimentation on a testbed of Linux machines running Java to study the effect of immediate role assignment and the time complexity of optimised role assignment. At the beginning of the experiment the number and types of roles are changed in the mission class specification and the requisite number of instances of the framework are started on various machines. The experimental setup consisted of machines $^{2}$ on the Local Area Network ${ }^{3}$. We simulated different subnets by using IP filter policies. Each manager role was assigned to a separate machine and a different subnet, while other roles were running in parallel (with a maximum of 20 roles per machine). Some earlier results are available in [13,14]. In a real system, wireless networks would be used rather than Local Area Networks (LANs) with a subnet corresponding to nodes within a single hop broadcast range. We checked policy load times in the above experimental network and these were linear with respect to the number of policies, as expected.

\subsection{Comparison of the Immediate and Optimal Role Assignment Approaches}

In this experiment we compare the performance of the immediate and optimal role assignments with respect to time complexity and success rate of assignment. We considered a mission which has 10 role types and 10 UAV types as shown in Table 1. The capabilities of the first half of the UAV types, i.e. $s_{1}$

\footnotetext{
2 Intel(R) Core(TM)2 Duo CPU 3.00GHz, 4GB RAM.

$31 \mathrm{~Gb}$ ethernet.
} 


\begin{tabular}{|c|c|c|c|}
\hline $\begin{array}{l}\text { Role } \\
\text { Type }\end{array}$ & $\begin{array}{l}\text { Required } \\
\text { Capability }\end{array}$ & $\begin{array}{l}\text { UAV } \\
\text { Type }\end{array}$ & $\begin{array}{l}\text { Provided } \\
\text { Capability }\end{array}$ \\
\hline$r_{1}$ & $\left\{c_{1}, c_{2}\right\}$ & $s_{1}$ & $\left\{c_{1}, c_{2}\right\}$ \\
\hline$r_{2}$ & $\left\{c_{1}, c_{3}\right\}$ & $s_{2}$ & $\left\{c_{1}, c_{3}\right\}$ \\
\hline$r_{3}$ & $\left\{c_{1}, c_{4}\right\}$ & $s_{3}$ & $\left\{c_{1}, c_{4}\right\}$ \\
\hline$r_{4}$ & $\left\{c_{1}, c_{5}\right\}$ & $s_{4}$ & $\left\{c_{1}, c_{5}\right\}$ \\
\hline$r_{5}$ & $\left\{c_{1}, c_{6}\right\}$ & $s_{5}$ & $\left\{c_{1}, c_{6}\right\}$ \\
\hline$r_{6}$ & $\left\{c_{1}, c_{7}\right\}$ & $s_{6}$ & $\left\{c_{1}, c_{2}, c_{3}, c_{4}, c_{5}, c_{6}, c_{7}\right\}$ \\
\hline$r_{7}$ & $\left\{c_{8}\right\}$ & $s_{7}$ & $\left\{c_{1}, c_{2}, c_{3}, c_{4}, c_{5}, c_{6}, c_{8}\right\}$ \\
\hline$r_{8}$ & $\left\{c_{9}\right\}$ & $s_{8}$ & $\left\{c_{1}, c_{2}, c_{3}, c_{4}, c_{5}, c_{6}, c_{9}\right\}$ \\
\hline$r_{9}$ & $\left\{c_{10}\right\}$ & $s_{9}$ & $\left\{c_{1}, c_{2}, c_{3}, c_{4}, c_{5}, c_{6}, c_{10}\right\}$ \\
\hline$r_{10}$ & $\left\{c_{11}\right\}$ & $s_{10}$ & $\left\{c_{1}, c_{2}, c_{3}, c_{4}, c_{5}, c_{6}, c_{11}\right\}$ \\
\hline
\end{tabular}

Table 1 Capability Requirements and Provisions

up to $s_{5}$, can each satisfy one role type (one out of $r_{1}$ up to $r_{5}$ ). Each of the second half of the UAV types ( $s_{6}$ up to $s_{10}$ ) has capabilities which are diverse enough to satisfy the capability requirement of any of the first five role types $\left(r_{1}\right.$ up to $\left.r_{5}\right)$ as well as one other role type out of the second half of the role types $\left(r_{6}\right.$ up to $\left.r_{10}\right)$. Each of the first five role types can be assigned to one of the 6 possible UAV types while each of the rest of the role types can only be assigned to one UAV type.

If we perform an immediate role assignment on a first-discovered firstassigned basis, it may be the case that one or more of role types $r_{1}$ up to $r_{5}$ are assigned to a UAV of type $s_{6}$ up to $s_{10}$ leading to an incomplete team since role types $r_{6}$ up to $r_{10}$ can only be assigned to UAV types $s_{6}$ up to $s_{10}$ respectively. The team can be complete only if the UAVs arrive in a sequence where all the first half of UAV types arrive before any of the UAV types of the second half, i.e. $s_{1}, s_{2}, s_{3}, s_{4}, s_{5} \rightarrow s_{6}, s_{7}, s_{8}, s_{9}, s_{10}$. There are 5 ! $* 5$ ! possibilities of this arrival out of 10 ! possible arrival sequences. In the worst case scenario all of the second half of the UAV types can arrive first leading to a team where only $50 \%$ of the roles are assigned.

In our experiment we varied the number of roles and UAVs keeping their numbers equal and the role and UAV types and their proportions constant. In the case of the optimised role assignment all the roles were assigned in all the experiments. Fig. 23 shows the result of the immediate role assignment for 20 experiments plotted with a $95 \%$ confidence interval where Role Assignment Success Rate is the percentage of roles assigned to UAVs, out of the total number of available roles. The plot labelled as Immediate shows the observed success rate of the immediate role assignment scheme during the experiment. Note that the success rate of this assignment scheme varies as it depends on UAV arrival sequences. The plot labelled as Optimised shows the observed success rate of the optimised role assignment scheme. Theoretically this success 
rate can also be achieved in the immediate role assignment scheme (best case scenario) provided that the UAVs arrive in an ideal sequence, i.e. all the first half of UAV types (shown in Table 1) arrive before any of the UAV types of the second half which is impractical. The plot labelled as Immediate (worst case) shows what the success rate would be if the UAVs arrive in the worst possible sequence, i.e. all of the second half of the UAV types (shown in Table 1) arrive before the first half.
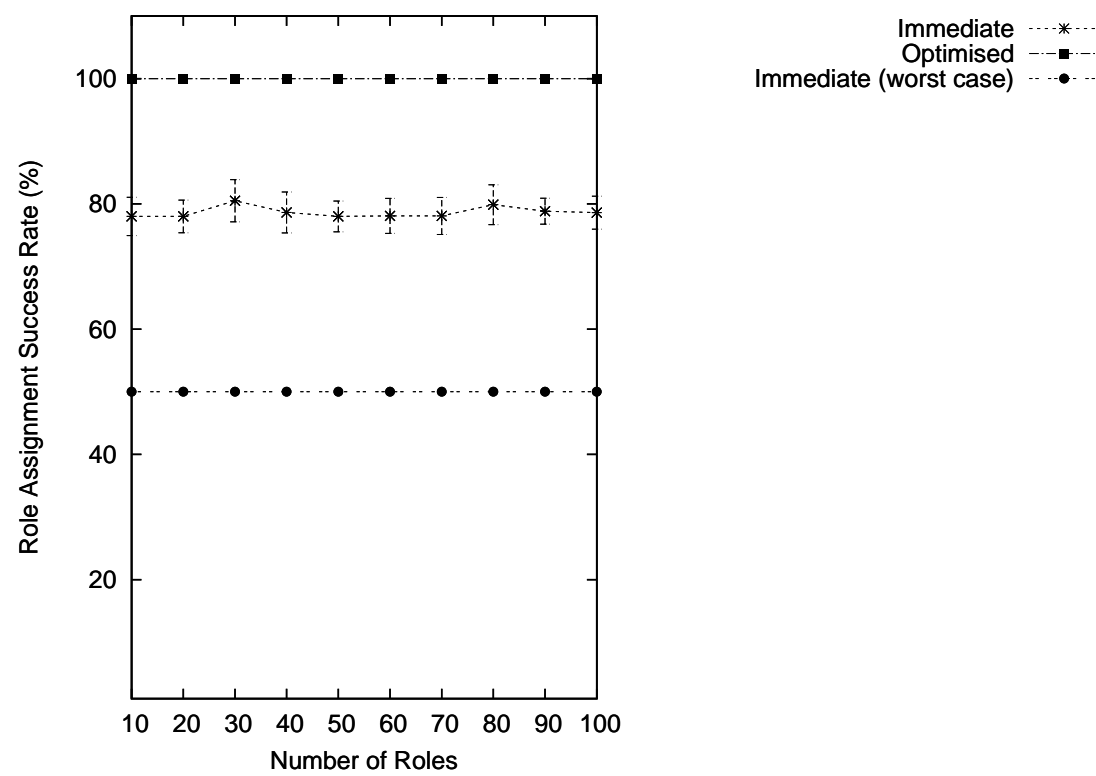

Fig. 23 Role Assignment Success Rate

Fig. 24 shows the time taken for assignment for both immediate and optimised role assignments. The assignment time is measured from the time the first UAV is discovered to either the time the last role is assigned (in the case of the optimised assignment) or all UAVs have been discovered and all roles that can be assigned are assigned (in the case of the immediate assignment). The polynomial time complexity of the optimised assignment is attributed to the $O\left(n^{3}\right)$ algorithm ${ }^{4}$ we used to compute the minimum-cost maximum bipartite matching of the assignment graph. Although the assignment algorithm, for the sake of the experiment, starts optimisation after all UAVs are discovered, in reality the waiting period is set by a policy and there is a tradeoff between the length of this period and the success rate of the assignment.

\footnotetext{
${ }^{4} \mathrm{n}$ is the number of roles/UAVs.
} 


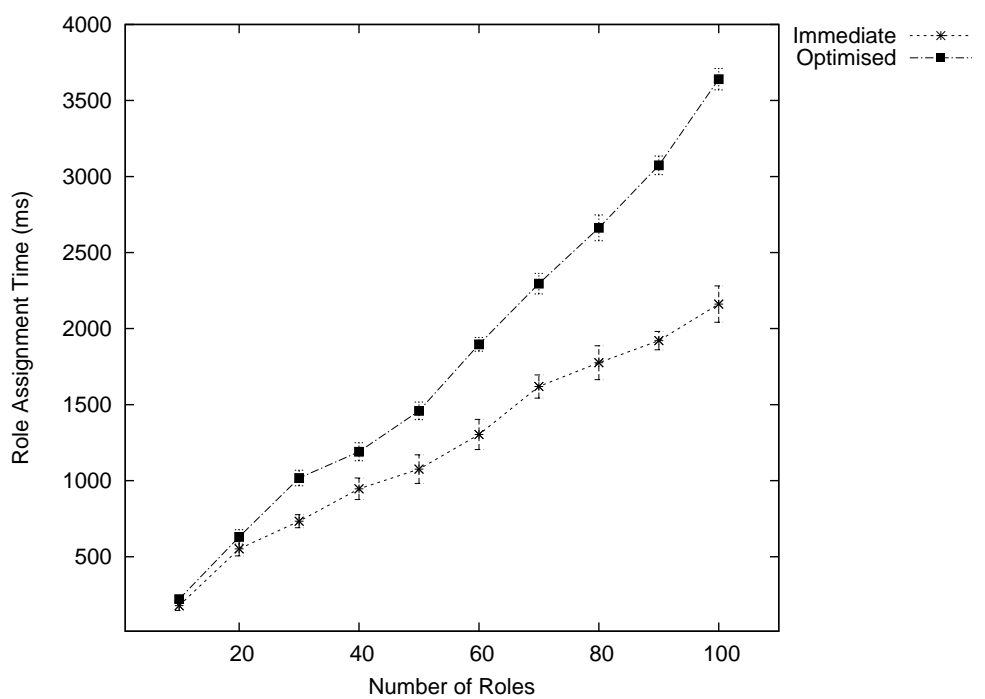

Fig. 24 Time Complexity of the Optimised and Immediate Assignment Algorithms

\section{Related Work}

Specification of the organisation of a set of primitives to obtain a sophisticated mission to perform complex tasks is presented in [32]. This includes a Configuration Description Language (CDL) to configure a single or group of robots by defining reusable assemblage agents for different tasks and instantiate the primitives. The MissionLab development environment supports graphical design of a mission using CDL, compilation and loading executable code onto a robot or a simulator. However, the finite state machine based approach used to describe a mission is suitable only for low level components such as tasks and it is not easy to specify multi-robot missions with many participants.

A case based reasoning approach for generating mission plans [40] builds on the work in [32] and uses a Contract Net Protocol [37] based task allocation while [37] presents the Contract Net Protocol for distributing tasks through negotiation. Each node in the net takes either a manager or contractor role. Managers announce tasks, potential contractors submit bids to the managers, the managers then evaluate the bids and award contracts to the bidders.

An approach for coordination of robots based on dynamic role assignment is given in [20]. This has a layered architecture with a coordination protocol, based on utility functions defined for each role, using a publish-subscribe communication protocol. The robot with the highest role utility value is assigned to the role. Formation is selected using a voting system. Compared to other approaches (e.g., [34]), which tie the robot control architecture to the coordination architecture (mechanism), this is more general in that robots with different control architectures can coordinate. 
A paradigm for cooperating robots is presented in [11] in which hybrid automata are used to represent roles, role assignments and discrete variables related to each robot. The composition of these automata is used to model the execution of cooperative tasks. A role is defined as a function one or more robots perform during the execution of a cooperative task and utility functions are used to decide when to change roles.

Likhachev et al. [28] have proposed an approach to automatic modification of behavioural assemblage parameters for autonomous navigation tasks using case based reasoning. We try to solve a more generic problem using policies.

In [18] the authors present an approach for exploration, mapping and tracking targets using large scale heterogeneous robots. The failure detection approach is slightly similar to our approach in that the leader robot detecting the failure of the follower is comparable to a parent role detecting the failure of its child. However, their approach to recovery is coded in the behaviour of the robots while we use policies for managing failure.

Jamp [42] uses disconnected operations to handle communication link disconnections and defines an abstraction called container in order to facilitate the implementation of mobile applications. An application in Jamp is implemented as an interaction between containers, since containers can be moved from node to node. The container concept in the Jamp system and its mobility is similar to our role concept. However, Jamp is not applicable for communication link failures since it is not possible to transfer state information to the newly instantiated container in another node. Our approach caters for link disconnections by periodically collecting state information.

Our role assignment approach which deals with assigning roles to different autonomous systems in an optimal manner is related to the more general optimal system deployment and reconfiguration problem.

In $[3,2]$ the dynamic reconfiguration process is perceived as the sense-planact process in robot control and the planning part of this process is addressed using an LPG planner and a tool referred to as Planit which provides the domain and configuration specifications specified using PDDL, to the planner. The planner generates sequences of actions to lead the system to the desired state, these are passed to Planit which performs the actions on the system. Planit monitors the system and obtains events from which it generates the current state of the system. Reconfiguration can be used to improve the quality of deployment as well as for failure recovery.

In $[21,24]$ a framework that consists of a declarative service specification in terms of components, support for component deployment and a planning module is presented. It enables services to be built up from distributed components and facilitates migration and replication of components transparently. The service specification and the current state of the network is provided to the planner [24] which determines optimal locations for component placement. The planning module employs multiple planning techniques in that it uses regression and progression planning. The framework assumes that the network on which the distributed system is deployed is static with respect of the set of nodes and links as well as their properties. 
COCoA [25] formulates the problem of task allocation and scheduling for a tightly coupled (i.e. no robot can achieve a single goal by itself) team of robots, in a search and rescue domain, using goals and constraints. Three constraints namely goal, robot and resource constraints are specified using first order logic. The constraint optimisation problem is modelled as a Mixed Integer Linear Programming (MILP) problem and CPLEX [19] is used to solve it. Since finding a feasible solution might take a considerable amount of time (hours, even days for large search and rescue problems), different heuristics are used to improve the solution time.

\section{Conclusion}

In this paper we have presented a policy-based self-management architecture for mobile autonomous systems which has three layers namely mission, team and communication. The mission layer deals with specification of missions in terms of roles. In order to allow adaptation of missions and reuse of specifications, the mission is specified in three levels, namely mission class, mission instance and policy specifications. In accordance to the mission specification, the team layer forms teams dynamically by discovering UAVs using a discovery service and admitting them to the team securely. Using a public key infrastructure, UAVs are authenticated and a secure channel is created if the authentication succeeds. A policy-based optimisation is used to perform optimal role assignment. The optimisation is utility based and it allows for adding new utility functions and changing the behaviour of the existing ones using policies. The team responds to intermittent communication link and permanent UAV failures using the failure management protocol.

Communication among team members is maintained by the communication layer which controls the movement of the UAVs in order to keep them within the communication link range of each other. Should the prevention of communication link maintenance fails, a rendezvous is set up to facilitate intermittent communication.

Although we have focussed on UAVs, the techniques described in this paper can be applied to manage any mobile, team-based collaboration such as is found in military missions, search and rescue or disaster relief scenarios. In these applications team members may change frequently, and tasks also need to be assigned based on capabilities.

We have evaluated the performance of our architecture with respect to different performance measures including scalability and time complexity and observed that it performs well.

\section{Acknowledgements}

The work reported in this paper was funded by the Systems Engineering for Autonomous Systems (SEAS) Defence Technology Centre established by the UK Ministry of Defence and UK EPSRC Aedus2 project (Grant EP/E025188/1). 


\section{References}

1. H. Ando, Y. Oasa, I. Suzuki, and M. Yamashita. Distributed memoryless point convergence algorithm for mobile robots with limited visibility. IEEE Transactions on Robotics and Automation, 15(5):818-828, Oct 1999.

2. N. Arshad, D. Heimbigner, and A.L. Wolf. Deployment and dynamic reconfiguration planning for distributed software systems. Software Quality Journal, 15(3):265-281, 2007.

3. Naveed Arshad, Dennis Heimbigner, and Alexander. L. Wolf. Deployment and dynamic reconfiguration planning for distributed software systems. In ICTAI '03: Proceedings of the 15th IEEE International Conference on Tools with Artificial Intelligence, page 39, Washington, DC, USA, 2003. IEEE Computer Society.

4. Eskindir Asmare and Morris Sloman. Self-management Framework for Unmanned Autonomous Vehicles. In Inter-Domain Management, First International Conference on Autonomous Infrastructure, Management and Security, volume 4543 of Lecture Notes in Computer Science, pages 164-167, July 2007.

5. E. Bicho and S. Monteiro. Formation control for multiple mobile robots: A non-linear attractor dynamics approach. In Proceedings IEEE/RSJ International Conference on Intelligent Robots and Systems, 2003.

6. Burton H. Bloom. Space/time trade-offs in hash coding with allowable errors. Commun. ACM, 13(7):422-426, 1970.

7. François Bourgeois and Jean-Claude Lassalle. An extension of the munkres algorithm for the assignment problem to rectangular matrices. Commun. ACM, 14(12):802-804, 1971 .

8. A. Broder and M. Mitzenmacher. Network applications of bloom filters: A survey. Internet Mathematics, 1(4):485-509, 2004.

9. J. Carlson and RR Murphy. How UGVs physically fail in the field. IEEE Transactions on Robotics, 21(3):423-437, 2005.

10. G. Carpaneto, S. Martello, and P. Toth. Algorithms and codes for the assignment problem. Annals of Operations Research, 13(1):191-223, 1988.

11. L.T.R. Chaimowicz, V.T.R. Kumar, and M.F.M.T.R. Campos. A paradigm for dynamic coordination of multiple robots. Autonomous Robots, 17(1):7-21, 2004.

12. W. Diffie and M. Hellman. New directions in cryptography. IEEE Transactions on Information Theory, 22(6):644-654, 1976.

13. E. Asmare, A. Gopalan, M. Sloman, N. Dulay and E. C. Lupu. A Policy-Based Management Architecture for Mobile Collaborative Teams. In Proceedings of the 7th Annual IEEE International Conference on Pervasive Computing and Communications, 2009.

14. E. Asmare, A. Gopalan, M. Sloman, N. Dulay and E. Lupu. A Mission Management Framework for Unmanned Autonomous Vehicles. In Proceedings of the The Second International ICST Conference on MOBILe Wireless MiddleWARE, Operating Systems, and Applications, 2009.

15. J.E. Hopcroft and R.M. Karp. An $n^{5 / 2}$ Algorithm for Maximum Matchings in Bipartite Graphs. SIAM Journal on Computing, 2:225, 1973.

16. P. Horn. Autonomic computing: IBMs perspective on the state of information technology. IBM Corporation, October 2001.

17. R. Housley et al. Internet X. 509 Public Key Infrastructure Certificate and CRL Profile. Technical report, RFC 2459, January, 1999.

18. Andrew Howard et al. The sdr experience: Experiments with a large-scale heterogenous mobile robot team. In 9th International Symposium on Experimental Robotics, 2004.

19. C.O. Inc. CPLEX Linear Optimizer and Mixed Integer Optimizer. Suite, 279:930, 2003.

20. L.B. Iocchi, D.B. Nardi, M.B. Piaggio, and A.B. Sgorbissa. Distributed coordination in heterogeneous multi-robot systems. Autonomous Robots, 15(2):155-168, 2003.

21. A.A. Ivan, J. Harman, M. Allen, and V. Karamcheti. Partitionable services: A framework for seamlessly adapting distributed applications to heterogeneous environments. In 11th IEEE International Symposium on High Performance Distributed Computing, 2002. HPDC-11 2002. Proceedings, pages 103-112, 2002.

22. Jeffrey O. Kephart and David M. Chess. The vision of autonomic computing. IEEE Computer, 36(1):41-50, 2003. 
23. JO Kephart and WE Walsh. An artificial intelligence perspective on autonomic computing policies. Policies for Distributed Systems and Networks, 2004. POLICY 2004. Proceedings. Fifth IEEE International Workshop on, pages 3-12, 2004.

24. T. Kichkaylo, A. Ivan, and V. Karamcheti. Constrained component deployment in widearea networks using AI planning techniques. In Parallel and Distributed Processing Symposium, 2003. Proceedings. International, page 10, 2003.

25. M. Koes, I. Nourbakhsh, and K. Sycara. Constraint optimization coordination architecture for search and rescue robotics. In Robotics and Automation, 2006. ICRA 2006. Proceedings 2006 IEEE International Conference on, pages 3977-3982, May 2006.

26. H. Kuhn. Variants of the Hungarian method for assignment problems. Naval Research Logistics Quarterly, 3:253-258, 1956.

27. H. W Kuhn. The Hungarian Method For The Assignment Problem1. Naval research logistics quarterly, page 83, 1955.

28. Maxim Likhachev, Michael Kaess, Zsolt Kira, and Ronald C. Arkin. Spatio-temporal case-based reasoning for efficient reactive robot navigation. Mobile Robot Labaratory, Georgia Institute of Technology, 2005.

29. J. Lin, A. S. Morse, and B. D. O. Anderson. The multi-agent rendezvous problem. part 1: The synchronous case. SIAM J. Control Optim., 46(6):2096-2119, 2007.

30. E. Lupu et al. AMUSE: Autonomic management of ubiquitous e-health systems. Concurrency and Computation: Practice \& Experience, 20(3):277-295, 2008.

31. T. Bourdenas M. Sloman and E. C. Lupu. Self-healing for Pervasive Computing Systems. In Architecture Dependable Systems VII, edited by Rogerio de Lemos et. al, to be published, Lecture Notes in Computer Science, 2010.

32. D.C. MacKenzie, R. Arkin, and J.M. Cameron. Multiagent mission specification and execution. Autonomous Robots, 4(1):29-52, 1997.

33. James Munkres. Algorithms for the assignment and transportation problems. Journal of the Society for Industrial and Applied Mathematics, 5(1):32-38, 1957.

34. LE Parker. ALLIANCE: An architecture for fault tolerant multirobot cooperation. IEEE Transactions on Robotics and Automation, 14(2):220-240, 1998

35. Ponder2. http://ponder2.net, 2008.

36. Alberto Schaeffer-Filho et al. Towards supporting interactions between self-managed cells. In Proceedings of the First IEEE International Conference on Self-Adaptive and Self-Organizing Systems, pages 224-233, Boston, MA, USA, 2007.

37. R.G. Smith. The Contract Net Protocol: High-level communication and control in a distributed problem solver. IEEE Transactions on Computers, C-29(12), 1980.

38. Ichiro Suzuki and Masafumi Yamashita. Distributed anonymous mobile robots: Formation of geometric patterns. SIAM J. Comput., 28(4), 1999.

39. J. Sweeney, TJ Brunette, Y. Yang, and R. Grupen. Coordinated teams of reactive mobile platforms. In Proceedings of IEEE International Conference on Robotics and Automation, 2002.

40. P. Ulam, Y. Endo, A. Wagner, and R. Arkin. Integrated mission specification and task allocation for robot teams - design and implementation. IEEE International Conference on Robotics and Automation, pages 4428-4435, April 2007.

41. UpnP Forum. Upnp. Available at http://www.upnp.org (20/08/2009).

42. M. Valente, R. Bighonha, M. Bigonha, and A. Loureiro. Disconnected Operation in a Mobile Computation System. In Proceedings of the Workshop on Software Engineering and Mobility, 2001. 